\title{
Free Vibrations of a Cantilevered SWCNT with Distributed Mass in the Presence of Nonlocal Effect
}

\author{
M. A. De Rosa, ${ }^{1}$ M. Lippiello, ${ }^{2}$ and H. D. Martin $^{3}$ \\ ${ }^{1}$ School of Engineering, University of Basilicata, Viale dell'Ateneo Lucano 10, 85100 Potenza, Italy \\ ${ }^{2}$ Department of Structures for Engineering and Architecture, University of Naples "Federico II", Via Forno Vecchio 36, \\ 80134 Naples, Italy \\ ${ }^{3}$ Facultad Regional Reconquista, UTN, Parque Industrial Reconquista, Reconquista, 3560 Santa Fe, Argentina
}

Correspondence should be addressed to M. A. De Rosa; maria.derosa@unibas.it

Received 7 October 2014; Accepted 19 December 2014

Academic Editor: Sayan Bhattacharyya

Copyright (c) 2015 M. A. De Rosa et al. This is an open access article distributed under the Creative Commons Attribution License, which permits unrestricted use, distribution, and reproduction in any medium, provided the original work is properly cited.

\begin{abstract}
The Hamilton principle is applied to deduce the free vibration frequencies of a cantilever single-walled carbon nanotube (SWCNT) in the presence of an added mass, which can be distributed along an arbitrary part of the span. The nonlocal elasticity theory by Eringen has been employed, in order to take into account the nanoscale effects. An exact formulation leads to the equations of motion, which can be solved to give the frequencies and the corresponding vibration modes. Moreover, two approximate semianalytical methods are also illustrated, which can provide quick parametric relationships. From a more practical point of view, the problem of detecting the mass of the attached particle has been solved by calculating the relative frequency shift due to the presence of the added mass: from it, the mass value can be easily deduced. The paper ends with some numerical examples, in which the nonlocal effects are thoroughly investigated.
\end{abstract}

\section{Introduction}

Carbon nanotubes (CNT) - as discovered by Iijima in 1991 (see [1])-have unique electrical, mechanical, and thermal properties, so that they are widely used in a large range of technical areas: nanoelectronics, scanning probes, nanoscale sensors, biomedical devices, and others. From a theoretical point of view, the nanoscale of these structures suggests an atomistic model, but this approach turns out to be very expensive. On the other hand, the usual beam theories (EulerBernoulli, Timoshenko, or even higher-order theories [2]) do not capture the influence of the size-effects, because they are inherently scale-free, so that it is usual to adopt the nonlocal elasticity theory, as developed by Eringen in [3, 4]. One of the most important goals of the nanomechanics is to use biosensors in order to detect external deposited masses, and a good mechanical model can be assumed to be a cantilever beam with an attached added mass along the span. It is important to note that the usual hypothesis of a "point mass" is not always justified [5-11], whereas a more realistic model [12] should assume a distributed added mass along a finite portion of the span. For this model, the free vibration frequencies can be calculated according to the classical energy method: the equations of motion and the boundary conditions are derived by applying the Hamilton principle, and the resulting boundary value problem is solved, to give the secular equation, which in turn permits deducing the frequencies.

Sometimes it is necessary to deduce the influence of some control parameter on the free vibration frequencies, so that some parametric curves must be sketched: in these cases the so-called semianalytical (SAN) methods become unvaluable, because they lead to approximate closed-form formulae for the frequencies as functions of the control parameter. In this paper, the added mass will be treated as a control parameter, and the use of two approximate approaches will permit us to examine the variation of the first frequency as a function of the added mass. In the first approach, we generalize a Meirovitch suggestion [13], starting from the equations of motion, in the spirit of Galerkin, whereas in the second method we start from the energies, following a Ritzlike approach. Both methods give close approximations to 


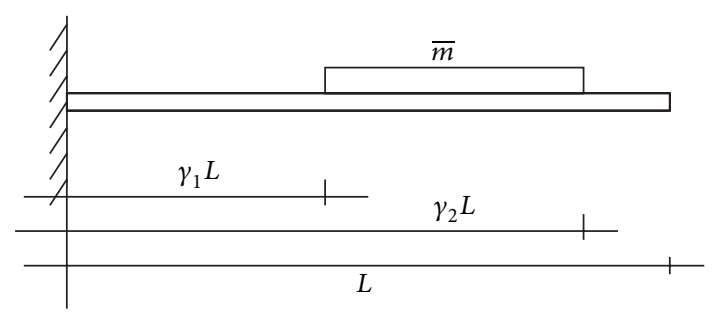

FIGURE 1: Geometrical properties of the nanotube.

the true results, so that it is possible to use the parametric curve in order to find the added mass in terms of the frequency shift.

\section{Analysis of the Problem}

Let us consider the cantilevered nanotube in Figure 1, with span $L$, cross-sectional area $A$, second moment of area $I$, and mass density $\rho$. The well-known Euler-Bernoulli theory for slender beams will be used, so that the Young modulus $E$ suffices to define the material properties. Finally, an attached distributed mass $\bar{m}$ is located, between the abscissae $\gamma_{1} L$ and $\gamma_{2} L$. In order to take into account the nanoscale effects, the nonlocal elasticity theory has been adopted, as suggested by Eringen.

According to Hamilton principle it is possible to write

$$
\int_{t_{1}}^{t_{2}}\left(\delta T(t)-\delta E_{T}(t)\right) \mathrm{dt}=0,
$$

where

$$
\begin{aligned}
T= & \frac{1}{2} \int_{0}^{\gamma_{1} L} \rho A\left(\frac{\partial v_{1}(z, t)}{\partial t}\right)^{2} \mathrm{~d} z+\frac{1}{2} \int_{\gamma_{1} L}^{\gamma_{2} L} \rho A\left(\frac{\partial v_{2}(z, t)}{\partial t}\right)^{2} \mathrm{~d} z \\
& +\frac{1}{2} \int_{\gamma_{2} L}^{L} \rho A\left(\frac{\partial v_{3}(z, t)}{\partial t}\right)^{2} \mathrm{~d} z+\frac{1}{2} \int_{\gamma_{1} L}^{\gamma_{2} L} \bar{m}\left(\frac{\partial v_{2}(z, t)}{\partial t}\right)^{2} \mathrm{~d} z
\end{aligned}
$$

is the sum of the kinetic energies of the nanotube in the three sections, $v_{1}$ between $\left(0, \gamma_{1} L\right), v_{2}$ between $\left(\gamma_{1} L, \gamma_{2} L\right)$, and $v_{3}$ between $\left(\gamma_{2} L, L\right)$, respectively, and of the kinetic energy of the added mass. Moreover,

$$
\begin{aligned}
E_{T}= & L_{e}-P=\frac{1}{2} \int_{0}^{\gamma_{1} L} \operatorname{EI}\left(\frac{\partial^{2} v_{1}(z, t)}{\partial z^{2}}\right)^{2} \mathrm{~d} z \\
& +\frac{1}{2} \int_{\gamma_{1} L}^{\gamma_{2} L} \operatorname{EI}\left(\frac{\partial^{2} v_{2}(z, t)}{\partial z^{2}}\right)^{2} \mathrm{~d} z \\
& +\frac{1}{2} \int_{\gamma_{2} L}^{L} \operatorname{EI}\left(\frac{\partial^{2} v_{3}(z, t)}{\partial z^{2}}\right)^{2} \mathrm{dz}
\end{aligned}
$$

$$
\begin{aligned}
& -\int_{0}^{\gamma_{1} L} \rho A \frac{\partial^{2} v_{1}(z, t)}{\partial t^{2}} \mu^{2} \frac{\partial^{2} v_{1}(z, t)}{\partial z^{2}} \mathrm{dz} \\
& -\int_{\gamma_{1} L}^{\gamma_{2} L} \rho A \frac{\partial^{2} v_{2}(z, t)}{\partial t^{2}} \mu^{2} \frac{\partial^{2} v_{2}(z, t)}{\partial z^{2}} \mathrm{dz} \\
& -\int_{\gamma_{2} L}^{L} \rho A \frac{\partial^{2} v_{3}(z, t)}{\partial t^{2}} \mu^{2} \frac{\partial^{2} v_{3}(z, t)}{\partial z^{2}} \mathrm{dz}
\end{aligned}
$$

is the total potential energy, $L_{e}$ is the strain energy of the nanotube, and $P$ is the potential energy of the inertial force $\left(\rho A\left(\partial^{2} v(z, t) / \partial t^{2}\right)\right)$ due to the additional displacement $\mu^{2}\left(\partial^{2} v(z, t) / \partial z^{2}\right)[14]$. Here $\mu^{2}=\left(e_{0} a\right)^{2}$, where $e_{0}$ is a material constant, which has to be defined through experimental results, and $a$ is the internal characteristic length of the nanotube.

The first variation of these two energies can be easily calculated so that (1) gives

$$
\begin{aligned}
& \int_{t_{1}}^{t_{2}}\left(\int_{0}^{\gamma_{1} L} \rho A \frac{\partial v_{1}(z, t)}{\partial t} \delta\left(\frac{\partial v_{1}(z, t)}{\partial t}\right) \mathrm{dz}\right. \\
& \quad+\int_{\gamma_{1} L}^{\gamma_{2} L} \rho A \frac{\partial v_{2}(z, t)}{\partial t} \delta\left(\frac{\partial v_{2}(z, t)}{\partial t}\right) \mathrm{d} z \\
& \quad+\int_{\gamma_{2} L}^{L} \rho A \frac{\partial v_{3}(z, t)}{\partial t} \delta\left(\frac{\partial v_{3}(z, t)}{\partial t}\right) \mathrm{d} z \\
& +\int_{\gamma_{1} L}^{\gamma_{2} L} \bar{m} \frac{\partial v_{2}(z, t)}{\partial t} \delta\left(\frac{\partial v_{2}(z, t)}{\partial t}\right) \mathrm{dz} \\
& \quad-\int_{0}^{\gamma_{1} L}\left(\mathrm{EI} \frac{\partial^{2} v_{1}(z, t)}{\partial z^{2}} \delta\left(\frac{\partial^{2} v_{1}(z, t)}{\partial z^{2}}\right)\right. \\
& \left.\quad-\mu^{2} \rho A \frac{\partial^{2} v_{1}(z, t)}{\partial t^{2}} \delta\left(\frac{\partial^{2} v_{1}(z, t)}{\partial z^{2}}\right)\right) \mathrm{d} z \\
& \quad-\int_{\gamma_{1} L}^{\gamma_{2} L}\left(\mathrm{EI} \frac{\partial^{2} v_{2}(z, t)}{\partial z^{2}} \delta\left(\frac{\partial^{2} v_{2}(z, t)}{\partial z^{2}}\right)\right. \\
& \left.-\mu^{2} \rho A \frac{\partial^{2} v_{2}(z, t)}{\partial t^{2}} \delta\left(\frac{\partial^{2} v_{2}(z, t)}{\partial z^{2}}\right)\right) \mathrm{d} z \\
& -\int_{\gamma_{2} L}^{L}\left(\mathrm{EI} \frac{\partial^{2} v_{3}(z, t)}{\partial z^{2}} \delta\left(\frac{\partial^{2} v_{3}(z, t)}{\partial z^{2}}\right)\right. \\
& \left.\left.-\mu^{2} \rho A \frac{\partial^{2} v_{3}(z, t)}{\partial t^{2}} \delta\left(\frac{\partial^{2} v_{3}(z, t)}{\partial z^{2}}\right)\right) \mathrm{d} z\right) \mathrm{dt}=0 .
\end{aligned}
$$

Integrations by part (see Appendix) lead to a system of three equations of motion:

$$
\begin{array}{r}
\operatorname{EI} \frac{\partial^{4} v_{1}(z, t)}{\partial z^{4}}-\mu^{2} \rho A \frac{\partial^{4} v_{1}(z, t)}{\partial z^{2} \partial t^{2}}+\rho A \frac{\partial^{2} v_{1}(z, t)}{\partial t^{2}}=0, \\
0<z<\gamma_{1} L,
\end{array}
$$




$$
\begin{gathered}
\mathrm{EI} \frac{\partial^{4} v_{2}(z, t)}{\partial z^{4}}-\mu^{2} \rho A \frac{\partial^{4} v_{2}(z, t)}{\partial z^{2} \partial t^{2}}+(\rho A+M) \frac{\partial^{2} v_{2}(z, t)}{\partial t^{2}}=0, \\
\gamma_{1} L<z<\gamma_{2} L, \\
\mathrm{EI} \frac{\partial^{4} v_{3}(z, t)}{\partial z^{4}}-\mu^{2} \rho A \frac{\partial^{4} v_{3}(z, t)}{\partial z^{2} \partial t^{2}}+\rho A \frac{\partial^{2} v_{3}(z, t)}{\partial t^{2}}=0, \\
\gamma_{2} L<z<L,
\end{gathered}
$$

together with the following general boundary conditions at the ends:

$$
\begin{gathered}
v_{1}(0, t)=0, \\
\frac{\partial v_{1}(0, t)}{\partial z}=0, \\
\mathrm{EI} \frac{\partial^{3} v_{3}(L, t)}{\partial z^{3}}-\mu^{2} \rho A \frac{\partial^{3} v_{3}(L, t)}{\partial t^{2} \partial z}=0, \\
-\mathrm{EI} \frac{\partial^{2} v_{3}(L, t)}{\partial z^{2}}+\mu^{2} \rho A \frac{\partial^{2} v_{3}(L, t)}{\partial t^{2}}=0 .
\end{gathered}
$$

The boundary conditions for $z=\gamma_{1} L$ are given by

$$
\begin{gathered}
v_{1}\left(\gamma_{1} L, t\right)=v_{2}\left(\gamma_{1} L, t\right), \\
\frac{\partial v_{1}\left(\gamma_{1} L, t\right)}{\partial z}=\frac{\partial v_{2}\left(\gamma_{1} L, t\right)}{\partial z}, \\
\mu^{2} \rho A \frac{\partial^{3} v_{1}\left(\gamma_{1} L, t\right)}{\partial t^{2} \partial z}-\mathrm{EI} \frac{\partial^{3} v_{1}\left(\gamma_{1} L, t\right)}{\partial z^{3}}-\mu^{2} \rho A \frac{\partial^{3} v_{2}\left(\gamma_{1} L, t\right)}{\partial t^{2} \partial z} \\
+\mathrm{EI} \frac{\partial^{3} v_{2}\left(\gamma_{1} L, t\right)}{\partial z^{3}}=0, \\
\mu^{2} \rho A \frac{\partial^{2} v_{1}\left(\gamma_{1} L, t\right)}{\partial t^{2}}-\mathrm{EI} \frac{\partial^{2} v_{1}\left(\gamma_{1} L, t\right)}{\partial z^{2}}-\mu^{2} \rho A \frac{\partial^{2} v_{2}\left(\gamma_{1} L, t\right)}{\partial t^{2}} \\
+\mathrm{EI} \frac{\partial^{2} v_{2}\left(\gamma_{1} L, t\right)}{\partial z^{2}}=0,
\end{gathered}
$$

and finally, at $z=\gamma_{2} L$,

$$
\begin{gathered}
v_{2}\left(\gamma_{2} L, t\right)=v_{3}\left(\gamma_{2} L, t\right), \\
\frac{\partial v_{2}\left(\gamma_{2} L, t\right)}{\partial z}=\frac{\partial v_{3}\left(\gamma_{2} L, t\right)}{\partial z}, \\
\mu^{2} \rho A \frac{\partial^{3} v_{2}\left(\gamma_{2} L, t\right)}{\partial t^{2} \partial z}-\mathrm{EI} \frac{\partial^{3} v_{2}\left(\gamma_{2} L, t\right)}{\partial z^{3}}-\mu^{2} \rho A \frac{\partial^{3} v_{3}\left(\gamma_{2} L, t\right)}{\partial t^{2} \partial z} \\
+\mathrm{EI} \frac{\partial^{3} v_{3}\left(\gamma_{2} L, t\right)}{\partial z^{3}}=0, \\
\mu^{2} \rho A \frac{\partial^{2} v_{2}\left(\gamma_{2} L, t\right)}{\partial t^{2}}-\mathrm{EI} \frac{\partial^{2} v_{2}\left(\gamma_{2} L, t\right)}{\partial z^{2}}-\mu^{2} \rho A \frac{\partial^{2} v_{3}\left(\gamma_{2} L, t\right)}{\partial t^{2}} \\
+\mathrm{EI} \frac{\partial^{2} v_{3}\left(\gamma_{2} L, t\right)}{\partial z^{2}}=0 .
\end{gathered}
$$

The solutions of (5) can be expressed as

$$
v_{h}(z, t)=v_{h}(z) e^{i \omega t}, \quad h=1,2,3 .
$$

If the nondimensional abscissa $\zeta=z / L$ can be introduced, the system of three equations of motion (5) becomes

$$
\begin{gathered}
\frac{\partial^{4} v_{1}(\zeta)}{\partial \zeta^{4}}+\eta^{2} \Omega^{4} \frac{\partial^{2} v_{1}(\zeta)}{\partial \zeta^{2}}-\Omega^{4} v_{1}(\zeta)=0 \quad \text { for } 0<\zeta<\gamma_{1}, \\
\frac{\partial^{4} v_{2}(\zeta)}{\partial \zeta^{4}}+\eta^{2} \Omega^{4} \frac{\partial^{2} v_{2}(\zeta)}{\partial \zeta^{2}}-(1+\lambda) \Omega^{4} v_{2}(\zeta)=0 \\
\text { for } \gamma_{1}<\zeta<\gamma_{2}, \\
\frac{\partial^{4} v_{3}(\zeta)}{\partial \zeta^{4}}+\eta^{2} \Omega^{4} \frac{\partial^{2} v_{3}(\zeta)}{\partial \zeta^{2}}-\Omega^{4} v_{3}(\zeta)=0 \quad \text { for } \gamma_{2}<\zeta<1,
\end{gathered}
$$

where the following nondimensional parameters have been introduced:

$$
\lambda=\frac{\bar{m}}{\rho A} ; \quad \eta=\frac{\mu}{L} ; \quad \Omega=\sqrt{\sqrt{\frac{\rho A L^{4} \omega^{2}}{\mathrm{EI}}}} .
$$

The boundary conditions for clamped-free nanotube are given by

$$
\begin{gathered}
v_{1}(0)=0, \\
\frac{\partial v_{1}(0)}{\partial \zeta}=0, \\
v_{1}\left(\gamma_{1}\right)=v_{2}\left(\gamma_{1}\right), \\
\frac{\partial v_{1}\left(\gamma_{1}\right)}{\partial \zeta}=\frac{\partial v_{2}\left(\gamma_{1}\right)}{\partial \zeta}, \\
\eta^{2} \Omega^{4} \frac{\partial v_{1}\left(\gamma_{1}\right)}{\partial \zeta}+\frac{\partial^{3} v_{1}\left(\gamma_{1}\right)}{\partial \zeta^{3}}-\eta^{2} \Omega^{4} \frac{\partial v_{2}\left(\gamma_{1}\right)}{\partial \zeta}-\frac{\partial^{3} v_{2}\left(\gamma_{1}\right)}{\partial \zeta^{3}}=0 \\
\eta^{2} \Omega^{4} v_{1}\left(\gamma_{1}\right)+\frac{\partial^{2} v_{1}\left(\gamma_{1}\right)}{\partial \zeta^{2}}-\eta^{2} \Omega^{4} v_{2}\left(\gamma_{1}\right)-\frac{\partial^{2} v_{2}\left(\gamma_{1}\right)}{\partial \zeta^{2}}=0 \\
v_{2}\left(\gamma_{2}\right)=v_{3}\left(\gamma_{2}\right), \\
\frac{\partial v_{2}\left(\gamma_{2}\right)}{\partial \zeta}=\frac{\partial v_{3}\left(\gamma_{2}\right)}{\partial \zeta}, \\
\eta^{2} \Omega^{4} \frac{\partial v_{2}\left(\gamma_{2}\right)}{\partial \zeta}+\frac{\partial^{3} v_{2}\left(\gamma_{2}\right)}{\partial \zeta^{3}}-\eta^{2} \Omega^{4} \frac{\partial v_{3}\left(\gamma_{2}\right)}{\partial \zeta}-\frac{\partial^{3} v_{3}\left(\gamma_{2}\right)}{\partial \zeta^{3}}=0 \\
\eta^{2} \Omega^{4} v_{2}\left(\gamma_{2}\right)+\frac{\partial^{2} v_{2}\left(\gamma_{2}\right)}{\partial \zeta^{2}}-\eta^{2} \Omega^{4} v_{3}\left(\gamma_{2}\right)-\frac{\partial^{2} v_{3}\left(\gamma_{2}\right)}{\partial \zeta^{2}}=0 \\
\frac{\partial^{3} v_{3}(1)}{\partial \zeta^{3}}+\eta^{2} \Omega^{4} \frac{\partial v_{3}(1)}{\partial \zeta}=0, \\
\eta^{2} \Omega^{4} v_{3}(1)=0 .
\end{gathered}
$$


The general solutions of (10) are given by

$$
\begin{aligned}
v_{1}(\zeta)= & A_{1} \cos (\alpha \zeta)+A_{2} \sin (\alpha \zeta)+A_{3} \cos h(\alpha \zeta) \\
& +A_{4} \sin h(\alpha \zeta) \\
v_{2}(\zeta)= & B_{1} \cos \left(\alpha_{1} \zeta\right)+B_{2} \sin \left(\alpha_{1} \zeta\right)+B_{3} \cos h\left(\beta_{1} \zeta\right) \\
& +B_{4} \sin h\left(\beta_{1} \zeta\right) \\
v_{3}(\zeta)= & C_{1} \cos (\alpha \zeta)+C_{2} \sin (\alpha \zeta)+C_{3} \cos h(\beta \zeta) \\
& +C_{4} \sin h(\beta \zeta)
\end{aligned}
$$

with

$$
\begin{aligned}
& \alpha=\sqrt{\frac{1}{2}\left(\eta^{2} \Omega^{4}+\Omega^{2} \sqrt{4+\eta^{4} \Omega^{4}}\right)} ; \\
& \beta=\sqrt{\frac{1}{2}\left(-\eta^{2} \Omega^{4}+\Omega^{2} \sqrt{4+\eta^{4} \Omega^{4}}\right)} ; \\
& \alpha_{1}=\sqrt{\frac{1}{2}\left(\eta^{2} \Omega^{4}+\Omega^{2} \sqrt{4+4 \lambda+\eta^{4} \Omega^{4}}\right)} ; \\
& \beta_{1}=\sqrt{\frac{1}{2}\left(-\eta^{2} \Omega^{4}+\Omega^{2} \sqrt{4+4 \lambda+\eta^{4} \Omega^{4}}\right)} .
\end{aligned}
$$

The twelve constants can be found by imposing the boundary conditions (12). The resulting homogeneous system has nontrivial solutions if and only if the coefficient determinant is zero and the corresponding secular equation has infinite solutions $\Omega_{j}$. The circular frequencies $\omega_{j}$ can be easily deduced, as well as the natural frequencies $f_{j}=\omega_{j} / 2 \pi$.

2.1. Nonlocal Fundamental Natural Frequency on CNT with Attached Mass-First Method. Starting from the equations of motion (5), it is possible to integrate each of them in their domain, and the resulting integrals can be summed up:

$$
\begin{aligned}
\int_{0}^{L} \mathrm{EI} & \frac{\partial^{4} v(z)}{\partial z^{4}} \mathrm{~d} z+\omega^{2} \int_{0}^{L} \mu^{2} \rho A \frac{\partial^{2} v(z)}{\partial z^{2}} \mathrm{dz} \\
& -\omega^{2} \int_{0}^{L} \rho A v(z) \mathrm{d} z-\omega^{2} \int_{\gamma_{1} L}^{\gamma_{2} L} \bar{m} v(z) \mathrm{d} z=0 .
\end{aligned}
$$

It is now possible to insert a trial function $y(z)$ [14], leading to

$$
\begin{aligned}
\int_{0}^{L} \mathrm{EI} & \frac{\partial^{4} v(z)}{\partial z^{4}} y(z) \mathrm{d} z+\omega^{2} \int_{0}^{L} \mu^{2} \rho A \frac{\partial^{2} v(z)}{\partial z^{2}} y(z) \mathrm{d} z \\
& -\omega^{2} \int_{0}^{L} \rho A v(z) y(z) \mathrm{d} z-\omega^{2} \int_{\gamma_{1} L}^{\gamma_{2} L} \bar{m} v(z) y(z) \mathrm{d} z=0 .
\end{aligned}
$$

Two successive integrations by part can be performed:

$$
\begin{aligned}
\int_{0}^{L} \mathrm{EI} & \frac{\partial^{4} v(z)}{\partial z^{4}} y(z) \mathrm{dz} \\
= & {\left[\mathrm{EI} \frac{\partial^{3} v(z)}{\partial z^{3}} y(z)\right]_{0}^{L}-\int_{0}^{L} \mathrm{EI} \frac{\partial^{3} v(z)}{\partial z^{3}} \frac{\partial y(z)}{\partial z} \mathrm{~d} z } \\
= & {\left[\mathrm{EI} \frac{\partial^{3} v(z)}{\partial z^{3}} y(z)\right]_{0}^{L}-\left[\mathrm{EI} \frac{\partial^{2} v(z)}{\partial z^{2}} \frac{\partial y(z)}{\partial z}\right]_{0}^{L} } \\
& +\int_{0}^{L} \mathrm{EI} \frac{\partial^{2} v(z)}{\partial z^{2}} \frac{\partial^{2} y(z)}{\partial z^{2}} \mathrm{dz},
\end{aligned}
$$

$$
\begin{aligned}
\omega^{2} \int_{0}^{L} & \mu^{2} \rho A \frac{\partial^{2} v(z)}{\partial z^{2}} y(z) \mathrm{d} z \\
= & {\left[\omega^{2} \mu^{2} \rho A \frac{\partial v(z)}{\partial z} y(z)\right]_{0}^{L}-\omega^{2} \int_{0}^{L} \mu^{2} \rho A \frac{\partial v(z)}{\partial z} \frac{\partial y(z)}{\partial z} \mathrm{~d} z } \\
= & {\left[\omega^{2} \mu^{2} \rho A \frac{\partial v(z)}{\partial z} y(z)\right]_{0}^{L}-\left[\omega^{2} \mu^{2} \rho A v(z) \frac{\partial y(z)}{\partial z}\right]_{0}^{L} } \\
& +\omega^{2} \int_{0}^{L} \mu^{2} \rho A v(z) \frac{\partial^{2} y(z)}{\partial z^{2}} \mathrm{dz}
\end{aligned}
$$

so that (16) becomes

$$
\begin{aligned}
\int_{0}^{L} \mathrm{EI} & \frac{\partial^{2} v(z)}{\partial z^{2}} \frac{\partial^{2} y(z)}{\partial z^{2}} \mathrm{~d} z-\omega^{2} \int_{0}^{L} \rho A v(z) y(z) \mathrm{d} z \\
& -\omega^{2} \int_{\gamma_{1} L}^{\gamma_{2} L} \bar{m} v(z) y(z) \mathrm{d} z+\omega^{2} \int_{0}^{L} \mu^{2} \rho A v(z) \frac{\partial^{2} y(z)}{\partial z^{2}} \mathrm{~d} z \\
+ & {\left[\mathrm{EI} \frac{\partial^{3} v(z)}{\partial z^{3}} y(z)\right]_{0}^{L}-\left[\mathrm{EI} \frac{\partial^{2} v(z)}{\partial z^{2}} \frac{\partial y(z)}{\partial z}\right]_{0}^{L} } \\
+ & {\left[\omega^{2} \mu^{2} \rho A \frac{\partial v(z)}{\partial z} y(z)\right]_{0}^{L}-\left[\omega^{2} \mu^{2} \rho A v(z) \frac{\partial y(z)}{\partial z}\right]_{0}^{L}=0 . }
\end{aligned}
$$

The boundary conditions at the right end permit simplifying the previous equation:

$$
\begin{aligned}
\int_{0}^{L} \mathrm{EI} & \frac{\partial^{2} v(z)}{\partial z^{2}} \frac{\partial^{2} y(z)}{\partial z^{2}} \mathrm{~d} z+\omega^{2} \int_{0}^{L} \mu^{2} \rho A v(z) \frac{\partial^{2} y(z)}{\partial z^{2}} \mathrm{~d} z \\
& -\omega^{2} \int_{0}^{L} \rho A v(z) y(z) \mathrm{d} z-\omega^{2} \int_{\gamma_{1} L}^{\gamma_{2} L} \bar{m} v(z) y(z) \mathrm{d} z \\
& +\mathrm{EI} \frac{\partial^{3} v(L)}{\partial z^{3}} y(L)-\mathrm{EI} \frac{\partial^{2} v(L)}{\partial z^{2}} \frac{\partial y(L)}{\partial z} \\
& +\omega^{2} \mu^{2} \rho A \frac{\partial v(L)}{\partial z} y(L)-\omega^{2} \mu^{2} \rho A v(L) \frac{\partial y(L)}{\partial z}=0
\end{aligned}
$$


whereas the free end will be subjected to the following equilibrium conditions:

$$
\begin{aligned}
& \omega^{2} \mu^{2} \rho A \frac{\partial v(L)}{\partial z}+\mathrm{EI} \frac{\partial^{3} v(L)}{\partial z^{3}}=0 \\
& -\omega^{2} \mu^{2} \rho A v(L)-\mathrm{EI} \frac{\partial^{2} v(L)}{\partial z^{2}}=0 .
\end{aligned}
$$

Finally, (19) reduces to

$$
\begin{aligned}
\int_{0}^{L} \mathrm{EI} & \frac{\partial^{2} v(z)}{\partial z^{2}} \frac{\partial^{2} y(z)}{\partial z^{2}} \mathrm{~d} z+\omega^{2} \int_{0}^{L} \mu^{2} \rho A v(z) \frac{\partial^{2} y(z)}{\partial z^{2}} \mathrm{~d} z \\
& -\omega^{2} \int_{0}^{L} \rho A v(z) y(z) \mathrm{d} z-\omega^{2} \int_{\gamma_{1} L}^{\gamma_{2} L} \bar{m} v(z) y(z) \mathrm{d} z=0
\end{aligned}
$$

and the frequency $\omega^{2}$ can be written down, putting $y(z)=$ $v(z)$, as

$$
\begin{aligned}
\omega^{2}= & \left(\int_{0}^{L} \mathrm{EI} \frac{\partial^{2} v(z)}{\partial z^{2}} \frac{\partial^{2} v(z)}{\partial z^{2}} \mathrm{dz}\right) \\
& \times\left(\int_{0}^{L} \rho A v^{2}(z) \mathrm{d} z+\int_{\gamma_{1} L}^{\gamma_{2} L} \bar{m} v^{2}(z) \mathrm{d} z\right. \\
& \left.-\int_{0}^{L} \mu^{2} \rho A v(z) \frac{\partial^{2} v(z)}{\partial z^{2}} \mathrm{dz}\right)^{-1}
\end{aligned}
$$

or, in terms of the nondimensional abscissa $\zeta=z / L$,

$$
\begin{aligned}
\omega^{2}= & \left(\frac{\mathrm{EI}}{L^{3}} \int_{0}^{1} \frac{\partial^{2} v(\zeta)}{\partial \zeta^{2}} \frac{\partial^{2} v(\zeta)}{\partial \zeta^{2}} \mathrm{~d} \zeta\right) \\
& \times\left(\rho A L \int_{0}^{1} v^{2}(\zeta) \mathrm{d} \zeta+\bar{m} L \int_{\gamma_{1}}^{\gamma_{2}} v^{2}(\zeta) \mathrm{d} \zeta\right. \\
& \left.-\eta^{2} \rho A L \int_{0}^{1} v(\zeta) \frac{\partial^{2} v(\zeta)}{\partial \zeta^{2}} \mathrm{~d} \zeta\right)^{-1} .
\end{aligned}
$$

In order to obtain a satisfactory approximation of the fundamental frequency, we use as approximating function $v(\zeta)$ the exact displacement of the cantilever beam without added mass:

$$
\begin{aligned}
v(\zeta)= & (\operatorname{Cosh}(1.8751 \zeta)-\operatorname{Cos}(1.8751 \zeta)) \\
& -\frac{(\operatorname{Sinh}(1.8751)-\operatorname{Sin}(1.8751))}{(\operatorname{Cosh}(1.8751)+\operatorname{Cos}(1.8751))} \\
& \times(\operatorname{Sinh}(1.8751 \zeta)-\operatorname{Sin}(1.8751 \zeta))
\end{aligned}
$$

The following integrals can be defined [15]:

$$
\begin{aligned}
& I_{1}=\int_{0}^{1} v^{2}(\zeta) \mathrm{d} \zeta=1 \\
& I_{3}=\int_{0}^{1} \frac{\partial^{2} v(\zeta)}{\partial \zeta^{2}} \frac{\partial^{2} v(\zeta)}{\partial \zeta^{2}} \mathrm{~d} \zeta=12.3623
\end{aligned}
$$

For the case of distributed added mass between the abscissae $\gamma_{1} L$ and $\gamma_{2} L$, the integral $I_{2}$ can be defined as

$$
I_{2}=\int_{\gamma_{1}}^{\gamma_{2}} v^{2}(\zeta) \mathrm{d} \zeta \quad 0 \leq \gamma_{1} \leq 1 ; 0 \leq \gamma_{2} \leq 1,
$$

and finally, in order to take into account the nonlocal effects, we define the fourth integral:

$$
I_{4}=\int_{0}^{1} v(\zeta) \frac{\partial^{2} v(\zeta)}{\partial \zeta^{2}} \mathrm{~d} \zeta=0.858264
$$

The fundamental natural frequency can be deduced from (23) in terms of these four integrals as

$$
f_{\mathrm{n} 1}=\frac{\omega}{2 \pi}=\frac{\beta}{2 \pi} \sqrt{\frac{I_{3}}{I_{1}+\lambda I_{2}-\eta^{2} I_{4}}} .
$$

Finally, it is usual to cancel out the first integral, so arriving to the natural frequency,

$$
f_{\mathrm{n} 1}=\frac{\beta}{2 \pi} \frac{C_{k}}{\sqrt{1+\lambda C_{m}-\eta^{2} C_{\mathrm{n} 1}}},
$$

where

$$
\begin{aligned}
\beta & =\sqrt{\frac{\mathrm{EI}}{m L^{4}}} ; \\
C_{k} & =\sqrt{\frac{I_{3}}{I_{1}}}=3.5160 ; \\
C_{\mathrm{n} 1} & =\frac{I_{4}}{I_{1}}=0.858264 ; \\
C_{m} & =\frac{I_{2}}{I_{1}}
\end{aligned}
$$

and $C_{k}, C_{\mathrm{n} 1}$, and $C_{m}$ are the so-called calibration constants.

2.2. Nonlocal Fundamental Natural Frequency on CNT with Attached Mass-Second Method. In this approach, let us start from the energy terms:

$$
\begin{aligned}
T=\frac{1}{2} \int_{0}^{L} \rho A\left(\frac{\partial v(z, t)}{\partial t}\right)^{2} \mathrm{~d} z+\frac{1}{2} \int_{\gamma_{1} L}^{\gamma_{2} L} \bar{m}\left(\frac{\partial v(z, t)}{\partial t}\right)^{2} \mathrm{~d} z \\
E_{T}=L_{e}-P \\
=\frac{1}{2} \int_{0}^{L} \mathrm{EI}\left(\frac{\partial^{2} v(z)}{\partial z^{2}}\right)^{2} \mathrm{~d} z \\
-\int_{0}^{L} \mu^{2} \rho A \frac{\partial^{2} v(z, t)}{\partial t^{2}} \frac{\partial^{2} v(z, t)}{\partial z^{2}} \mathrm{dz}
\end{aligned}
$$

and let us assume the separation of variables

$$
v(z, t)=v(z) \operatorname{Cos}(\omega \mathrm{t})
$$


so that the energies read

$$
\begin{aligned}
T= & \frac{\omega^{2}}{2}\left(\int_{0}^{L} \rho A v(z)^{2} \mathrm{~d} z+\int_{\gamma_{1} L}^{\gamma_{2} L} \bar{m} v(z)^{2} \mathrm{~d} z\right) \operatorname{Cos}^{2}(\omega t) \\
E_{T}= & L_{e}-P \\
= & \frac{1}{2}\left(\int_{0}^{L} \operatorname{EI}\left(\frac{\partial^{2} v(z)}{\partial z^{2}}\right)^{2} \mathrm{~d} z\right) \operatorname{Sin}^{2}(\omega t) \\
& +\omega^{2} \int_{0}^{L}\left(\mu^{2} \rho A v(z)\left(\frac{\partial^{2} v(z)}{\partial z^{2}}\right) \mathrm{d} z\right) \operatorname{Sin}^{2}(\omega t)
\end{aligned}
$$

The maximum kinetic energy will be equal to the maximum total potential energy, so that

$$
\begin{gathered}
\frac{1}{2} \int_{0}^{L} \operatorname{EI}\left(\frac{\partial^{2} v(z)}{\partial z^{2}}\right)^{2} \mathrm{~d} z+\omega^{2} \int_{0}^{L} \mu^{2} \rho A v(z)\left(\frac{\partial^{2} v(z)}{\partial z^{2}}\right) \mathrm{d} z \\
=\frac{\omega^{2}}{2} \int_{0}^{L} \rho A v(z)^{2} \mathrm{~d} z+\frac{\omega^{2}}{2} \int_{\gamma_{1} L}^{\gamma_{2} L} \bar{m} v(z)^{2} \mathrm{~d} z
\end{gathered}
$$

and the frequency $\omega^{2}$ can be deduced as

$$
\begin{aligned}
\omega^{2}= & \left(\frac{1}{2} \int_{0}^{L} \operatorname{EI}\left(\frac{\partial^{2} v(z)}{\partial z^{2}}\right)^{2} \mathrm{~d} z\right) \\
& \times\left(\frac{1}{2} \int_{0}^{L} \rho A v(z)^{2} \mathrm{~d} z+\frac{1}{2} \int_{\gamma_{1} L}^{\gamma_{2} L} \bar{m} v(z)^{2} \mathrm{~d} z\right. \\
& \left.-\int_{0}^{L} \mu^{2} \rho A v(z) \frac{\partial^{2} v(z)}{\partial z^{2}} \mathrm{~d} z\right)^{-1}
\end{aligned}
$$

$$
\begin{gathered}
\times\left(\frac{1}{2} \rho A L \int_{0}^{1} v^{2}(\zeta) \mathrm{d} \zeta+\frac{1}{2} \bar{m} L \int_{\gamma_{1}}^{\gamma_{2}} v^{2}(\zeta) \mathrm{d} \zeta\right. \\
\left.-\eta^{2} \rho A L \int_{0}^{1} v(\zeta) \frac{\partial^{2} v(\zeta)}{\partial \zeta^{2}} \mathrm{~d} \zeta\right)^{-1} .
\end{gathered}
$$

Let us assume the same approximating function equation (24), so that the following integrals can be calculated:

$$
\begin{aligned}
& I_{1}=\int_{0}^{1} v^{2}(\zeta) \mathrm{d} \zeta=1 \\
& I_{3}=\int_{0}^{1} \frac{\partial^{2} v(\zeta)}{\partial \zeta^{2}} \frac{\partial^{2} v(\zeta)}{\partial \zeta^{2}} \mathrm{~d} \zeta=12.3623 \\
& I_{4}=\int_{0}^{1} v(\zeta) \frac{\partial^{2} v(\zeta)}{\partial \zeta^{2}} \mathrm{~d} \zeta=0.858264
\end{aligned}
$$

and finally if the added mass is placed between the abscissae $\gamma_{1} L$ and $\gamma_{2} L$, the integral $I_{2}$ can be obtained as

$$
I_{2}=\int_{\gamma_{1}}^{\gamma_{2}} v^{2}(\zeta) \mathrm{d} \zeta ; \quad 0 \leq \gamma_{1} \leq 1 ; 0 \leq \gamma_{2} \leq 1 .
$$

Therefore, an alternative version of the natural frequency can be obtained as

$$
f_{\mathrm{n} 2}=\frac{\beta}{2 \pi} \frac{C_{k}}{\sqrt{1+\lambda C_{m}-\eta^{2} C_{\mathrm{n} 2}}}
$$

with the three calibration constants:

$$
\begin{aligned}
C_{k} & =\sqrt{\frac{I_{3}}{I_{1}}}=3.51601 ; \\
C_{\mathrm{n} 2} & =\frac{2 I_{4}}{I_{1}}=1.71653 ; \\
C_{m} & =\frac{I_{2}}{I_{1}} .
\end{aligned}
$$

\section{Nonlocal Sensor Equations}

The sensor equations in the presence of nonlocal elasticity can now be deduced, and the added mass $\bar{m}$ of a biomolecule can be detected by calculating the corresponding CNT frequency shift. In fact, let us start from the natural frequency of the CNT without the added mass:

$$
f_{\mathrm{n} 0}=\frac{1}{2 \pi} C_{k} \beta,
$$

and let us express the natural frequency, in the presence of the added mass, as (cf. (29) and (39))

$$
f_{\mathrm{ni}}=\frac{f_{\mathrm{n} 0}}{\sqrt{\left(1+\lambda C_{m}-\eta^{2} C_{\mathrm{ni}}\right)}}, \quad \mathrm{i}=1,2 .
$$

The frequency shift of the biosensor can be defined as

$$
\Delta \mathrm{f}=f_{\mathrm{n} 0}-f_{\mathrm{ni}}
$$

and finally the relative frequency shift is given by

$$
\frac{\Delta \mathrm{f}}{f_{\mathrm{n} 0}}=\left(1-\frac{1}{\sqrt{ }\left(1+\lambda C_{m}-\eta^{2} C_{\mathrm{ni}}\right)}\right)
$$

from which the value of the added mass $\bar{m}$ can be easily obtained:

$$
\begin{gathered}
\left(\frac{\Delta \mathrm{f}}{f_{\mathrm{n} 0}}-1\right)^{2}=\frac{1}{\left(1+\lambda C_{m}-\eta^{2} C_{\mathrm{ni}}\right)}, \\
\lambda=\frac{1}{C_{m}\left(\Delta \mathrm{f} / f_{\mathrm{n} 0}-1\right)^{2}}+\frac{C_{\mathrm{ni}} \eta^{2}}{C_{m}}-\frac{1}{C_{m}} \\
\bar{m}=\frac{\rho A}{C_{m}\left(\Delta \mathrm{f} / f_{\mathrm{n} 0}-1\right)^{2}}+\frac{C_{\mathrm{ni}} \eta^{2} \rho A}{C_{m}}-\frac{\rho A}{C_{m}}
\end{gathered}
$$

and $i=1$ for the first approach and $i=2$ for the second approach. 
TABLE 1: Nanotube properties (see [16]).

\begin{tabular}{lccc}
\hline $\begin{array}{l}\text { SWCNT } \\
\text { properties } \\
\text { density }\end{array}$ & Symbol & Value & Unit \\
\hline $\begin{array}{l}\text { Cross section } \\
\text { area }\end{array}$ & $A$ & $7.85110^{-19}$ & $\mathrm{~m}^{2}$ \\
$\begin{array}{l}\text { Radius } \\
\begin{array}{l}\text { Length } \\
\text { Moment of }\end{array}\end{array}$ & $R$ & $0.510^{-9}$ & $\mathrm{~m}$ \\
$\begin{array}{l}\text { inertia } \\
\text { Density }\end{array}$ & $I$ & $910^{-9}$ & $\mathrm{~m}$ \\
$\begin{array}{l}\text { Young's } \\
\text { modulus }\end{array}$ & $\rho$ & $4.9110^{-38}$ & $\mathrm{~m}^{4}$ \\
\hline
\end{tabular}

TABLE 2: The first exact natural frequency $\left(\times 10^{10}\right) f_{0}$ for various values of the nondimensional length of the added mass and for four increasing values of the nonlocal nondimensional coefficient $\eta$.

\begin{tabular}{lcccc}
\hline$\gamma$ & $\eta=0$ & $\eta=0.1$ & $\eta=0.3$ & $\eta=0.5$ \\
\hline 0.1 & 3.10361 & 3.11245 & 3.20756 & 3.46665 \\
0.2 & 2.84585 & 2.85418 & 2.9260 & 3.10933 \\
0.3 & 2.7036 & 2.71057 & 2.77009 & 2.91661 \\
0.4 & 2.62348 & 2.6297 & 2.68251 & 2.80985 \\
0.5 & 2.58023 & 2.58605 & 2.63527 & 2.75263 \\
0.6 & 2.55911 & 2.56473 & 2.6122 & 2.72473 \\
0.7 & 2.55048 & 2.55602 & 2.60277 & 2.71335 \\
0.8 & 2.54791 & 2.55342 & 2.59996 & 2.70995 \\
0.9 & 2.54749 & 2.55301 & 2.59951 & 2.70941 \\
\hline
\end{tabular}

\section{Numerical Examples}

4.1. First Example. Table 1 shows properties of the cantilever nanotube, which will be used throughout this section. The added distributed mass will be placed from the section $\gamma_{1} L$ to the free end, so that $\gamma_{2}=1$, and $\gamma_{1}$ will vary from 0.9 to 0.1 . In Table 2 the fundamental natural frequency is given for various values of the $\gamma=\gamma_{2}-\gamma_{1}=1-\gamma_{1}$ parameter and for increasing values of the nondimensional $\eta$ coefficient (see (11)). The first column gives the fundamental natural frequency in the absence of nonlocal effects. The table has been obtained by solving the system of three differential equations of motion (13), so that the results can be considered "exact." As can be easily observed, the first fundamental natural frequency increases for increasing values of the $\eta$ parameter, whereas it decreases for increasing values of the $\gamma$ parameter.

The fundamental natural frequency $f_{\mathrm{n} 1}$ is reported in Table 3, as obtained by means of (29) and with $\lambda=1$. A numerical comparison with the exact values in Table 1 shows that the relative error is greater for $\gamma=0.1$, whereas the results for $\gamma=0.9$ almost coincide. At $\gamma=0.1$, the relative error varies between $0.035 \%$ for $\eta=0.1,0.34 \%$ for $\eta=0.3$, and finally $2.36 \%$ for $\eta=0.5$. Of course, this $\eta$ value can be considered as a limiting case, whereas $\eta=0.1$ and $\eta=0.3$ are more realistic choices. For example (see [17]), $\eta=0.235$ is adopted. In Table 4 the fundamental natural frequency $f_{\mathrm{n} 2}$ is given, as obtained by means of (39) and with $\lambda=1$.
TABLE 3: First approximate fundamental natural frequency $\left(\times 10^{10}\right)$ $f_{\mathrm{nl}}$, as obtained using (29), for various values of the nondimensional length of the added mass and for four increasing values of the nonlocal nondimensional coefficient $\eta$.

\begin{tabular}{lcccc}
\hline$\gamma$ & $\eta=0$ & $\eta=0.1$ & $\eta=0.3$ & $\eta=0.5$ \\
\hline 0.1 & 3.10361 & 3.11354 & 3.19659 & 3.38478 \\
0.2 & 2.84803 & 2.85570 & 2.91937 & 3.06061 \\
0.3 & 2.70498 & 2.71154 & 2.76586 & 2.88508 \\
0.4 & 2.62411 & 2.63011 & 2.67959 & 2.78756 \\
0.5 & 2.580453 & 2.586150 & 2.63315 & 2.73539 \\
0.6 & 2.55916 & 2.56472 & 2.61054 & 2.71007 \\
0.7 & 2.55050 & 2.5560 & 2.60135 & 2.69979 \\
0.8 & 2.54792 & 2.55341 & 2.59861 & 2.69673 \\
0.9 & 2.54750 & 2.55299 & 2.59817 & 2.69624 \\
\hline
\end{tabular}

TABLE 4: First exact fundamental natural frequency $\left(\times 10^{10}\right) f_{\mathrm{n} 2}$, as obtained using (39), for various values of the nondimensional length of the added mass and for four increasing values of the nonlocal nondimensional coefficient $\eta$.

\begin{tabular}{lcccc}
\hline$\gamma$ & $\eta=0$ & $\eta=0.1$ & $\eta=0.3$ & $\eta=0.5$ \\
\hline 0.1 & 3.10361 & 3.12357 & 3.29845 & 3.75946 \\
0.2 & 2.84803 & 2.86343 & 2.99634 & 3.32922 \\
0.3 & 2.70498 & 2.71816 & 2.83106 & 3.10674 \\
0.4 & 2.62411 & 2.63614 & 2.73875 & 2.98593 \\
0.5 & 2.58045 & 2.59188 & 2.68921 & 2.92207 \\
0.6 & 2.55916 & 2.57032 & 2.66515 & 2.89127 \\
0.7 & 2.55050 & 2.56154 & 2.65536 & 2.87879 \\
0.8 & 2.54792 & 2.55893 & 2.65246 & 2.87509 \\
0.9 & 2.54750 & 2.55851 & 2.65199 & 2.87449 \\
\hline
\end{tabular}

A numerical comparison with the exact values in Table 2 shows that the relative error is greater than the previous case. More particularly, for $\gamma=0.1$ it varies between $0.36 \%$ for $\eta=0.1,2.83 \%$ for $\eta=0.3$, and finally $8.45 \%$ for $\eta=0.5$. Therefore, the first method seems to be more reliable than the second one.

4.2. Second Example. As a second example, let us suppose that the added mass is distributed along a fixed length, so that $\gamma=\gamma_{2}-\gamma_{1}=0.3$, but its real placement along the nanotube is unknown. In Table 5 the first fundamental natural frequency is reported, for different placements of the added mass and for four $\eta$ parameters. The frequencies have been obtained by solving the equations of motion, so that the results can be considered exact, and the nonlocal parameter has been allowed to vary between 0 and 0.3 . The fundamental natural frequency increases for increasing values of the nonlocal $\eta$ parameter, and higher values correspond to added masses nearer to the clamped end. The same example is illustrated in Tables 6 and 7, using the approximate formula (29) and the approximate formula (39), respectively. As in the first example, the first method gives better results.

4.3. Third Example. Finally, let us address the practical problem of the added mass detection. In order to solve this 
TABLE 5: First exact fundamental natural frequency $\left(\times 10^{10}\right) f_{0}$ for various values of the nondimensional length of the added mass (with $\gamma=0.3$ ) and for four increasing values of the nonlocal nondimensional coefficient $\eta$.

\begin{tabular}{cccccc}
\hline$\gamma_{1}$ & $\gamma_{2}$ & $\eta=0$ & $\eta=0.1$ & $\eta=0.2$ & $\eta=0.3$ \\
\hline 0.7 & 1 & 2.70498 & 2.71057 & 2.73205 & 2.77009 \\
0.6 & 0.9 & 2.90531 & 2.91317 & 2.9375 & 2.98072 \\
0.5 & 0.8 & 3.10026 & 3.10941 & 3.13780 & 3.18851 \\
0.4 & 0.7 & 3.27557 & 3.28637 & 3.31997 & 3.38053 \\
0.3 & 0.6 & 3.41695 & 3.42952 & 3.46879 & 3.54028 \\
0.2 & 0.5 & 3.51481 & 3.52888 & 3.57304 & 3.65415 \\
0.1 & 0.4 & 3.57016 & 3.58519 & 3.63251 & 3.71994 \\
0.0 & 0.3 & 3.59421 & 3.60970 & 3.65848 & 3.74886 \\
\hline
\end{tabular}

TABLE 6: First exact fundamental natural frequency $\left(\times 10^{10}\right) f_{1}$, as obtained using (29), for various values of the nondimensional length of the added mass (with $\gamma=0.3$ ) and for four increasing values of the nonlocal nondimensional coefficient $\eta$.

\begin{tabular}{cccccc}
\hline$\gamma_{1}$ & $\gamma_{2}$ & $\eta=0$ & $\eta=0.1$ & $\eta=0.2$ & $\eta=0.3$ \\
\hline 0.7 & 1 & 2.70498 & 2.71154 & 2.73154 & 2.76586 \\
0.6 & 0.9 & 2.90556 & 2.91370 & 2.93855 & 2.98142 \\
0.5 & 0.8 & 3.10177 & 3.11168 & 3.14201 & 3.19457 \\
0.4 & 0.7 & 3.27802 & 3.28973 & 3.32563 & 3.38815 \\
0.3 & 0.6 & 3.41894 & 3.43224 & 3.47306 & 3.54445 \\
0.2 & 0.5 & 3.51573 & 3.53019 & 3.57465 & 3.65264 \\
0.1 & 0.4 & 3.57039 & 3.585549 & 3.63215 & 3.71405 \\
0.0 & 0.3 & 3.59424 & 3.60969 & 3.65727 & 3.74092 \\
\hline
\end{tabular}

TABLE 7: First exact fundamental natural frequency $\left(\times 10^{10}\right) f_{2}$, as obtained using (39), for various values of the nondimensional length of the added mass (with $\gamma=0.3$ ) and for four increasing values of the nonlocal nondimensional coefficient $\eta$.

\begin{tabular}{cccccc}
\hline$\gamma_{1}$ & $\gamma_{2}$ & $\eta=0$ & $\eta=0.1$ & $\eta=0.2$ & $\eta=0.3$ \\
\hline 0.7 & 1 & 2.70498 & 2.71816 & 2.75890 & 2.83106 \\
0.6 & 0.9 & 2.90556 & 2.92191 & 2.97270 & 3.06355 \\
0.5 & 0.8 & 3.10177 & 3.12169 & 3.18385 & 3.29624 \\
0.4 & 0.7 & 3.27802 & 3.30157 & 3.37536 & 3.51017 \\
0.3 & 0.6 & 3.41894 & 3.44568 & 3.52982 & 3.68488 \\
0.2 & 0.5 & 3.51573 & 3.54482 & 3.63663 & 3.80690 \\
0.1 & 0.4 & 3.57039 & 3.60087 & 3.69723 & 3.87658 \\
0.0 & 0.3 & 3.59424 & 3.62534 & 3.72373 & 3.90716 \\
\hline
\end{tabular}

problem, it is necessary to plot the relationship between the added mass $\bar{m}$ equation (47) and the relative frequency shift equation (44). More precisely, in Figure 2 the nondimensional mass ratio $M / \rho \mathrm{AL}$ is plotted against the relative frequency shift equation (44), with $M=\bar{m}\left(\gamma_{2}-\gamma_{1}\right) L$, and the four curves refer to four different $\eta$ values, $\eta=0$ (without nonlocal effects), $\eta=0.1, \eta=0.2$, and $\eta=0.3$. The added mass is placed at the tip of the cantilever nanotube, so that $\eta_{2}=1$, whereas $\left(\gamma_{2}-\gamma_{1}\right)$ is allowed to vary between 0.05 and 0.6 . The geometrical data of the nanotube are given in Table 1. It is interesting to note that, according to our results, the relative

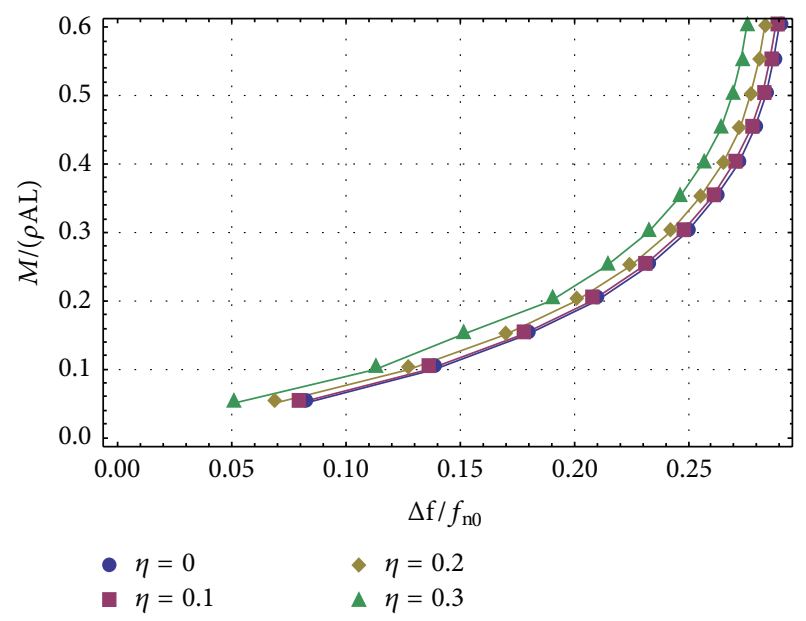

FIGURE 2: The nondimensional mass ratio $M / \rho \mathrm{AL}$ is plotted against the relative frequency shift-as obtained using (44)—with $M=$ $\bar{m}\left(\gamma_{2}-\gamma_{1}\right) L$. The four curves refer to four different $\eta$ values, $\eta=0$ (without nonlocal effects), $\eta=0.1, \eta=0.2$, and $\eta=0.3$.

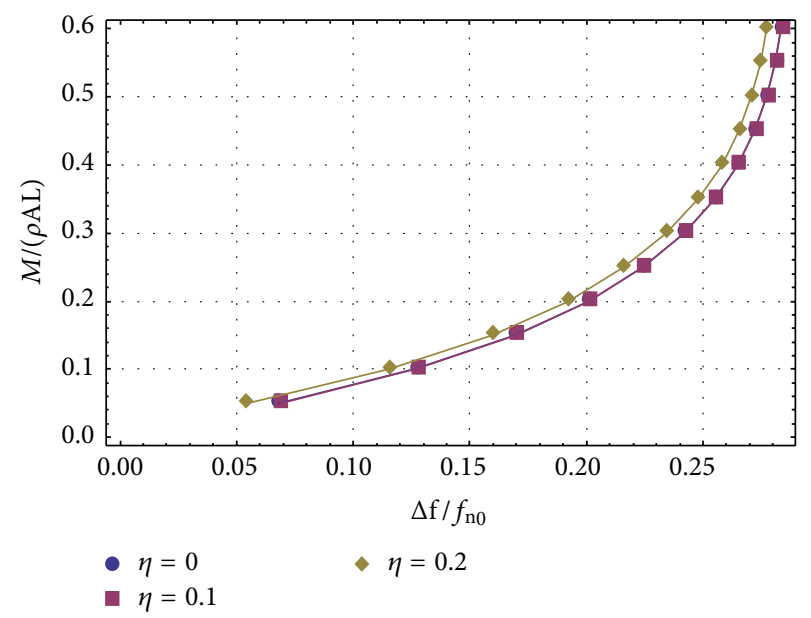

FIGURE 3: Numerical comparison between two proposed approaches.

frequency shift decreases for increasing values of the nonlocal coefficient $\eta$. This should be compared with the different behaviour exhibited by the results given in [17]. The curves in Figure 2 have been drawn using the first approach, because it gives better approximations to the true values. Actually, in Figure 3 we have compared the exact method with the two proposed approaches, for $\eta=0.2$, but the curve describing the first approach is undistinguishable from the exact curve.

\section{Conclusions}

The frequency shift between the free vibration frequencies of a cantilever nanotube with, and without, an attached distributed mass has been used, in order to detect the added mass value. It is shown that the size-effects must be taken into account, and the frequencies have to be calculated according to the nonlocal elasticity theory. Three different approaches 
have been proposed to solve the problem, and their results have been compared for a couple of examples. Moreover, the relative frequency shift decreases, for increasing values of the nonlocal coefficient $\eta$, so that careful calibration of this coefficient $\eta$ becomes necessary, in order to obtain reliable values of the added mass.

\section{Appendix}

Equation (4), which is reported here for the sake of readability, has to be integrated by part:

$$
\begin{gathered}
\int_{t_{1}}^{t_{2}}\left(\int_{0}^{\gamma_{1} L} \rho A \frac{\partial v_{1}(z, t)}{\partial t} \delta\left(\frac{\partial v_{1}(z, t)}{\partial t}\right) \mathrm{d} z\right. \\
+\int_{\gamma_{1} L}^{\gamma_{2} L} \rho A \frac{\partial v_{2}(z, t)}{\partial t} \delta\left(\frac{\partial v_{2}(z, t)}{\partial t}\right) \mathrm{d} z \\
+\int_{\gamma_{2} L}^{L} \rho A \frac{\partial v_{3}(z, t)}{\partial t} \delta\left(\frac{\partial v_{3}(z, t)}{\partial t}\right) \mathrm{d} z \\
+\int_{\gamma_{1} L}^{\gamma_{2} L} \bar{m} \frac{\partial v_{2}(z, t)}{\partial t} \delta\left(\frac{\partial v_{2}(z, t)}{\partial t}\right) \mathrm{d} z \\
-\int_{0}^{\gamma_{1} L}\left(\mathrm{EI} \frac{\partial^{2} v_{1}(z, t)}{\partial z^{2}} \delta\left(\frac{\partial^{2} v_{1}(z, t)}{\partial z^{2}}\right)\right. \\
-\int_{\gamma_{2} L}^{L}\left(\mathrm{EI} \frac{\partial^{2} v_{3}(z, t)}{\partial z^{2}} \delta\left(\frac{\partial^{2} v_{3}(z, t)}{\partial z^{2}}\right)\right. \\
\quad-\mu_{\gamma_{1} L}^{\gamma_{2} L}\left(\mathrm{EI} \frac{\partial^{2} v_{1}(z, t)}{\partial t^{2}} \delta\left(\frac{\partial^{2} v_{1}(z, t)}{\partial z^{2}}\right)\right) \mathrm{d} z \\
\quad \times z^{2} \rho\left(\frac{\partial^{2} v_{2}(z, t)}{\partial z^{2}}\right) \\
\left.\quad-\mu^{2} \rho A \frac{\partial^{2} v_{2}(z, t)}{\partial t^{2}} \delta\left(\frac{\partial^{2} v_{2}(z, t)}{\partial z^{2}}\right)\right) \mathrm{d} z
\end{gathered}
$$

The first three terms of (A.1) can be treated as follows:

$$
\begin{aligned}
\int_{0}^{\gamma_{1} L} \int_{t_{1}}^{t_{2}} \rho A & \frac{\partial v_{1}(z, t)}{\partial t} \delta\left(\frac{\partial v_{1}(z, t)}{\partial t}\right) \mathrm{dt} d z \\
= & \int_{0}^{\gamma_{1} L}\left[\rho A \frac{\partial v_{1}(z, t)}{\partial t} \delta\left(v_{1}(z, t)\right)\right]_{t_{1}}^{t_{2}} \mathrm{~d} z \\
& -\int_{0}^{\gamma_{1} L} \int_{t_{1}}^{t_{2}} \rho A \frac{\partial^{2} v_{1}(z, t)}{\partial t^{2}} \delta\left(v_{1}(z, t)\right) \mathrm{dt} \mathrm{d} z
\end{aligned}
$$

$$
\begin{gathered}
\int_{\gamma_{1} L}^{\gamma_{2} L} \int_{t_{1}}^{t_{2}} \rho A \frac{\partial v_{2}(z, t)}{\partial t} \delta\left(\frac{\partial v_{2}(z, t)}{\partial t}\right) \mathrm{dt} \mathrm{d} z \\
=\int_{\gamma_{1} L}^{\gamma_{2} L}\left[\rho A \frac{\partial v_{2}(z, t)}{\partial t} \delta\left(v_{2}(z, t)\right)\right]_{t_{1}}^{t_{2}} \mathrm{~d} z \\
-\int_{\gamma_{1} L}^{\gamma_{2} L} \int_{t_{1}}^{t_{2}} \rho A \frac{\partial^{2} v_{2}(z, t)}{\partial t^{2}} \delta\left(v_{2}(z, t)\right) \mathrm{dt} \mathrm{d} z \\
\int_{\gamma_{2} L}^{L} \int_{t_{1}}^{t_{2}} \rho A \frac{\partial v_{3}(z, t)}{\partial t} \delta\left(\frac{\partial v_{3}(z, t)}{\partial t}\right) \mathrm{dt} \mathrm{dz} \\
=\int_{\gamma_{2} L}^{L}\left[\rho A \frac{\partial v_{3}(z, t)}{\partial t} \delta\left(v_{3}(z, t)\right)\right]_{t_{1}}^{t_{2}} \mathrm{~d} z \\
-\int_{\gamma_{2} L}^{L} \int_{t_{1}}^{t_{2}} \rho A \frac{\partial^{2} v_{3}(z, t)}{\partial t^{2}} \delta\left(v_{3}(z, t)\right) \mathrm{dt} \mathrm{d} z
\end{gathered}
$$

where we considered the fact that $\delta\left(v_{i}(z, t)\right)=0$ at $t=t_{1}$ and $t=t_{2}$.

Quite similarly, the single term of the distributed mass becomes

$$
\begin{aligned}
\int_{\gamma_{1} L}^{\gamma_{2} L} \int_{t_{1}}^{t_{2}} \bar{m} \frac{\partial v_{2}(z, t)}{\partial t} \delta\left(\frac{\partial v_{2}(z, t)}{\partial t}\right) \mathrm{dt} d z \\
\quad=\int_{\gamma_{1} L}^{\gamma_{2} L}\left[\bar{m} \frac{\partial v_{2}(z, t)}{\partial t} \delta\left(v_{2}(z, t)\right)\right]_{t_{1}}^{t_{2}} \mathrm{dz} \\
\quad-\int_{\gamma_{1} L}^{\gamma_{2} L} \int_{t_{1}}^{t_{2}} \bar{m} \frac{\partial^{2} v_{2}(z, t)}{\partial t^{2}} \delta\left(v_{2}(z, t)\right) \mathrm{dt} \mathrm{d} z
\end{aligned}
$$

The nonlocal effects are contained into three integrals, which can be integrated as follows:

$$
\begin{aligned}
& \int_{t_{1}}^{t_{2}} \int_{0}^{\gamma_{1} L} \mu^{2} \rho A \frac{\partial^{2} v_{1}(z, t)}{\partial t^{2}} \delta\left(\frac{\partial^{2} v_{1}(z, t)}{\partial z^{2}}\right) \mathrm{dzdt} \\
& =\int_{t_{1}}^{t_{2}}\left[\mu^{2} \rho A \frac{\partial^{2} v_{1}(z, t)}{\partial t^{2}} \delta\left(\frac{\partial v_{1}(z, t)}{\partial z}\right)\right]_{0}^{\gamma_{1} L} \mathrm{dt} \\
& -\int_{t_{1}}^{t_{2}} \int_{0}^{\gamma_{1} L} \mu^{2} \rho A \frac{\partial^{3} v_{1}(z, t)}{\partial t^{2} \partial z} \delta\left(\frac{\partial v_{1}(z, t)}{\partial z}\right) \mathrm{dzdt} \\
& =\int_{t_{1}}^{t_{2}}\left[\mu^{2} \rho A \frac{\partial^{2} v_{1}(z, t)}{\partial t^{2}} \delta\left(\frac{\partial v_{1}(z, t)}{\partial z}\right)\right]_{0}^{\gamma_{1} L} \mathrm{dt} \\
& -\int_{t_{1}}^{t_{2}}\left[\mu^{2} \rho A \frac{\partial^{3} v_{1}(z, t)}{\partial t^{2} \partial z} \delta\left(v_{1}(z, t)\right)\right]_{0}^{\gamma_{1} L} \mathrm{dt} \\
& +\int_{t_{1}}^{t_{2}} \int_{0}^{\gamma_{1} L} \mu^{2} \rho A \frac{\partial^{4} v_{1}(z, t)}{\partial t^{2} \partial z^{2}} \delta\left(v_{1}(z, t)\right) \mathrm{d} z \mathrm{dt} ;
\end{aligned}
$$


10

The Scientific World Journal

$$
\begin{aligned}
& \int_{t_{1}}^{t_{2}} \int_{\gamma_{1} L}^{\gamma_{2} L} \mu^{2} \rho A \frac{\partial^{2} v_{2}(z, t)}{\partial t^{2}} \delta\left(\frac{\partial^{2} v_{2}(z, t)}{\partial z^{2}}\right) \mathrm{dzdt} \\
& =\int_{t_{1}}^{t_{2}}\left[\mu^{2} \rho A \frac{\partial^{2} v_{2}(z, t)}{\partial t^{2}} \delta\left(\frac{\partial v_{2}(z, t)}{\partial z}\right)\right]_{\gamma_{1} L}^{\gamma_{2} L} \mathrm{dt} \\
& -\int_{t_{1}}^{t_{2}} \int_{\gamma_{1} L}^{\gamma_{2} L} \mu^{2} \rho A \frac{\partial^{3} v_{2}(z, t)}{\partial t^{2} \partial z} \delta\left(\frac{\partial v_{2}(z, t)}{\partial z}\right) \mathrm{d} z \mathrm{dt} \\
& =\int_{t_{1}}^{t_{2}}\left[\mu^{2} \rho A \frac{\partial^{2} v_{2}(z, t)}{\partial t^{2}} \delta\left(\frac{\partial v_{2}(z, t)}{\partial z}\right)\right]_{\gamma_{1} L}^{\gamma_{2} L} \mathrm{dt} \\
& -\int_{t_{1}}^{t_{2}}\left[\mu^{2} \rho A \frac{\partial^{3} v_{2}(z, t)}{\partial t^{2} \partial z} \delta\left(v_{2}(z, t)\right)\right]_{\gamma_{1} L}^{\gamma_{2} L} \mathrm{dt} \\
& +\int_{t_{1}}^{t_{2}} \int_{\gamma_{1} L}^{\gamma_{2} L} \mu^{2} \rho A \frac{\partial^{4} v_{2}(z, t)}{\partial t^{2} \partial z^{2}} \delta\left(v_{2}(z, t)\right) \mathrm{dzdt} ; \\
& \int_{t_{1}}^{t_{2}} \int_{\gamma_{2} L}^{L} \mu^{2} \rho A \frac{\partial^{2} v_{3}(z, t)}{\partial t^{2}} \delta\left(\frac{\partial^{2} v_{3}(z, t)}{\partial z^{2}}\right) \mathrm{dzdt} \\
& =\int_{t_{1}}^{t_{2}}\left[\mu^{2} \rho A \frac{\partial^{2} v_{3}(z, t)}{\partial t^{2}} \delta\left(\frac{\partial v_{3}(z, t)}{\partial z}\right)\right]_{\gamma_{2} L}^{L} \mathrm{dt} \\
& -\int_{t_{1}}^{t_{2}} \int_{\gamma_{2} L}^{L} \mu^{2} \rho A \frac{\partial^{2} v_{3}(z, t)}{\partial t^{2}} \delta\left(\frac{\partial v_{3}(z, t)}{\partial z}\right) d z d t \\
& =\int_{t_{1}}^{t_{2}}\left[\mu^{2} \rho A \frac{\partial^{2} v_{3}(z, t)}{\partial t^{2}} \delta\left(\frac{\partial v_{3}(z, t)}{\partial z}\right)\right]_{\gamma_{2} L}^{L} \mathrm{dt} \\
& -\int_{t_{1}}^{t_{2}}\left[\mu^{2} \rho A \frac{\partial^{3} v_{3}(z, t)}{\partial t^{2} \partial z} \delta\left(v_{3}(z, t)\right)\right]_{\gamma_{2} L}^{L} \mathrm{dt} \\
& +\int_{t_{1}}^{t_{2}} \int_{\gamma_{2} L}^{L} \mu^{2} \rho A \frac{\partial^{4} v_{3}(z, t)}{\partial t^{2} \partial z^{2}} \delta\left(v_{3}(z, t)\right) \mathrm{d} z \mathrm{dt} .
\end{aligned}
$$

Finally, the first variation of the strain energy can be treated as usual:

$$
\begin{aligned}
-\int_{t_{1}}^{t_{2}} & \int_{0}^{\gamma_{1} L} \operatorname{EI} \frac{\partial^{2} v_{1}(z, t)}{\partial z^{2}} \delta\left(\frac{\partial^{2} v_{1}(z, t)}{\partial z^{2}}\right) \mathrm{d} z \mathrm{dt} \\
= & -\int_{t_{1}}^{t_{2}}\left[\mathrm{EI} \frac{\partial^{2} v_{1}(z, t)}{\partial z^{2}} \delta\left(\frac{\partial v_{1}(z, t)}{\partial z}\right)\right]_{0}^{\gamma_{1} L} \mathrm{dt} \\
& +\int_{t_{1}}^{t_{2}} \int_{0}^{\gamma_{1} L} \mathrm{EI} \frac{\partial^{3} v_{1}(z, t)}{\partial z^{3}} \delta\left(\frac{\partial v_{1}(z, t)}{\partial z}\right) \mathrm{dzdt} \\
= & -\int_{t_{1}}^{t_{2}}\left[\mathrm{EI} \frac{\partial^{2} v_{1}(z, t)}{\partial z^{2}} \delta\left(\frac{\partial v_{1}(z, t)}{\partial z}\right)\right]_{0}^{\gamma_{1} L} \mathrm{dt} \\
& +\int_{t_{1}}^{t_{2}}\left[\mathrm{EI} \frac{\partial^{3} v_{1}(z, t)}{\partial z^{3}} \delta\left(v_{1}(z, t)\right)\right]_{0}^{\gamma_{1} L} \mathrm{dt}
\end{aligned}
$$

$$
\begin{aligned}
& -\int_{t_{1}}^{t_{2}} \int_{0}^{\gamma_{1} L} \mathrm{EI} \frac{\partial^{4} v_{1}(z, t)}{\partial z^{4}} \delta\left(v_{1}(z, t)\right) \mathrm{d} z \mathrm{dt} ; \\
& -\int_{t_{1}}^{t_{2}} \int_{\gamma_{1} L}^{\gamma_{2} L} \operatorname{EI} \frac{\partial^{2} v_{2}(z, t)}{\partial z^{2}} \delta\left(\frac{\partial^{2} v_{2}(z, t)}{\partial z^{2}}\right) \mathrm{dzdt} \\
& =-\int_{t_{1}}^{t_{2}}\left[\mathrm{EI} \frac{\partial^{2} v_{2}(z, t)}{\partial z^{2}} \delta\left(\frac{\partial v_{2}(z, t)}{\partial z}\right)\right]_{\gamma_{1} L}^{\gamma_{2} L} \mathrm{dt} \\
& +\int_{t_{1}}^{t_{2}} \int_{\gamma_{1} L}^{\gamma_{2} L} \mathrm{EI} \frac{\partial^{3} v_{2}(z, t)}{\partial z^{3}} \delta\left(\frac{\partial v_{2}(z, t)}{\partial z}\right) \mathrm{d} z \mathrm{dt} \\
& =-\int_{t_{1}}^{t_{2}}\left[\mathrm{EI} \frac{\partial^{2} v_{2}(z, t)}{\partial z^{2}} \delta\left(\frac{\partial v_{2}(z, t)}{\partial z}\right)\right]_{\gamma_{1} L}^{\gamma_{2} L} \mathrm{dt} \\
& +\int_{t_{1}}^{t_{2}}\left[\mathrm{EI} \frac{\partial^{3} v_{2}(z, t)}{\partial z^{3}} \delta\left(v_{2}(z, t)\right)\right]_{\gamma_{1} L}^{\gamma_{2} L} \mathrm{dt} \\
& -\int_{t_{1}}^{t_{2}} \int_{\gamma_{1} L}^{\gamma_{2} L} \mathrm{EI} \frac{\partial^{4} v_{2}(z, t)}{\partial z^{4}} \delta\left(v_{2}(z, t)\right) \mathrm{dzdt} ; \\
& -\int_{t_{1}}^{t_{2}} \int_{\gamma_{2} L}^{L} \mathrm{EI} \frac{\partial^{2} v_{3}(z, t)}{\partial z^{2}} \delta\left(\frac{\partial^{2} v_{3}(z, t)}{\partial z^{2}}\right) \mathrm{dzdt} \\
& =-\int_{t_{1}}^{t_{2}}\left[\operatorname{EI} \frac{\partial^{2} v_{3}(z, t)}{\partial z^{2}} \delta\left(\frac{\partial v_{3}(z, t)}{\partial z}\right)\right]_{\gamma_{2} L}^{L} \mathrm{dt} \\
& +\int_{t_{1}}^{t_{2}} \int_{\gamma_{2} L}^{L} \mathrm{EI} \frac{\partial^{3} v_{3}(z, t)}{\partial z^{3}} \delta\left(\frac{\partial v_{3}(z, t)}{\partial z}\right) \mathrm{d} z \mathrm{dt} \\
& =-\int_{t_{1}}^{t_{2}}\left[\operatorname{EI} \frac{\partial^{2} v_{3}(z, t)}{\partial z^{2}} \delta\left(\frac{\partial v_{3}(z, t)}{\partial z}\right)\right]_{\gamma_{2} L}^{L} \mathrm{dt} \\
& +\int_{t_{1}}^{t_{2}}\left[\mathrm{EI} \frac{\partial^{3} v_{3}(z, t)}{\partial z^{3}} \delta\left(v_{3}(z, t)\right)\right]_{\gamma_{2} L}^{L} \mathrm{dt} \\
& -\int_{t_{1}}^{t_{2}} \int_{\gamma_{2} L}^{L} \mathrm{EI} \frac{\partial^{4} v_{3}(z, t)}{\partial z^{4}} \delta\left(v_{3}(z, t)\right) \mathrm{d} z \mathrm{dt} .
\end{aligned}
$$

All the previous integrated terms can be collected together, leading to

$$
\begin{aligned}
\int_{t_{1}}^{t_{2}}(- & \int_{0}^{\gamma_{1} L} \rho A \frac{\partial^{2} v_{1}(z, t)}{\partial t^{2}} \delta\left(v_{1}(z, t)\right) \mathrm{d} z \\
& -\int_{0}^{\gamma_{1} L} \mathrm{EI} \frac{\partial^{4} v_{1}(z, t)}{\partial z^{4}} \delta\left(v_{1}(z, t)\right) \mathrm{d} z \\
& +\int_{0}^{\gamma_{1} L} \mu^{2} \rho A \frac{\partial^{4} v_{1}(z, t)}{\partial t^{2} \partial z^{2}} \delta\left(v_{1}(z, t)\right) \mathrm{d} z \\
& -\int_{\gamma_{1} L}^{\gamma_{2} L} \rho A \frac{\partial^{2} v_{2}(z, t)}{\partial t^{2}} \delta\left(v_{2}(z, t)\right) \mathrm{d} z
\end{aligned}
$$




$$
\begin{aligned}
& -\int_{\gamma_{1} L}^{\gamma_{2} L} \operatorname{EI} \frac{\partial^{4} v_{2}(z, t)}{4} \delta\left(v_{2}(z, t)\right) \mathrm{d} z \\
& +\int_{\gamma_{1} L}^{\gamma_{2} L} \mu^{2} \rho A \frac{\partial^{4} v_{2}(z, t)}{\partial t^{2} \partial z^{2}} \delta\left(v_{2}(z, t)\right) \mathrm{d} z \\
& -\int_{\gamma_{2} L}^{L} \rho A \frac{\partial^{2} v_{3}(z, t)}{\partial t^{2}} \delta\left(v_{3}(z, t)\right) \mathrm{d} z \\
& -\int_{\gamma_{2} L}^{L} \operatorname{EI} \frac{\partial^{4} v_{3}(z, t)}{\partial z^{4}} \delta\left(v_{3}(z, t)\right) \mathrm{d} z \\
& +\int_{\gamma_{2} L}^{L} \mu^{2} \rho A \frac{\partial^{4} v_{3}(z, t)}{\partial t^{2} \partial z^{2}} \delta\left(v_{3}(z, t)\right) \mathrm{d} z \\
& \left.-\int_{\gamma_{1} L}^{\gamma_{2} L} \bar{m} \frac{\partial^{2} v_{2}(z, t)}{\partial t^{2}} \delta\left(v_{2}(z, t)\right) \mathrm{d} z\right) \mathrm{dt}=0 ; \\
& \int_{t_{1}}^{t_{2}}\left(\left[\mu^{2} \rho A \frac{\partial^{2} v_{1}(z, t)}{\partial t^{2}} \delta\left(\frac{\partial v_{1}(z, t)}{\partial z}\right)\right]_{0}^{\gamma_{1} L}\right. \\
& -\left[\mu^{2} \rho A \frac{\partial^{3} v_{1}(z, t)}{\partial t^{2} \partial z} \delta\left(v_{1}(z, t)\right)\right]_{0}^{\gamma_{1} L} \\
& -\left[\operatorname{EI} \frac{\partial^{2} v_{1}(z, t)}{\partial z^{2}} \delta\left(\frac{\partial v_{1}(z, t)}{\partial z}\right)\right]_{0}^{\gamma_{1} L} \\
& +\left[\mathrm{EI} \frac{\partial^{3} v_{1}(z, t)}{\partial z^{3}} \delta\left(v_{1}(z, t)\right)\right]_{0}^{\gamma_{1} L} \\
& +\left[\mu^{2} \rho A \frac{\partial^{2} v_{2}(z, t)}{\partial t^{2}} \delta\left(\frac{\partial v_{2}(z, t)}{\partial z}\right)\right]_{\gamma_{1} L}^{\gamma_{2} L} \\
& -\left[\mu^{2} \rho A \frac{\partial^{3} v_{2}(z, t)}{\partial t^{2} \partial z} \delta\left(v_{2}(z, t)\right)\right]_{\gamma_{1} L}^{\gamma_{2} L} \\
& -\left[\mathrm{EI} \frac{\partial^{2} v_{2}(z, t)}{\partial z^{2}} \delta\left(\frac{\partial v_{2}(z, t)}{\partial z}\right)\right]_{\gamma_{1} L}^{\gamma_{2} L} \\
& +\left[\mathrm{EI} \frac{\partial^{3} v_{2}(z, t)}{\partial z^{3}} \delta\left(v_{2}(z, t)\right)\right]_{\gamma_{1} L}^{\gamma_{2} L} \\
& +\left[\mu^{2} \rho A \frac{\partial^{2} v_{3}(z, t)}{\partial t^{2}} \delta\left(\frac{\partial v_{3}(z, t)}{\partial z}\right)\right]_{\gamma_{2} L}^{L} \\
& -\left[\mu^{2} \rho A \frac{\partial^{3} v_{3}(z, t)}{\partial t^{2} \partial z} \delta\left(v_{3}(z, t)\right)\right]_{\gamma_{2} L}^{L} \\
& -\left[\mathrm{EI} \frac{\partial^{2} v_{3}(z, t)}{\partial z^{2}} \delta\left(\frac{\partial v_{3}(z, t)}{\partial z}\right)\right]_{\gamma_{2} L}^{L} \\
& \left.+\left[\mathrm{EI} \frac{\partial^{3} v_{3}(z, t)}{\partial z^{3}} \delta\left(v_{3}(z, t)\right)\right]_{\gamma_{2} L}^{L}\right) \mathrm{dt}=0
\end{aligned}
$$

$$
\begin{gathered}
\mathrm{EI} \frac{\partial^{4} v_{1}(z, t)}{\partial z^{4}}-\mu^{2} \rho A \frac{\partial^{4} v_{1}(z, t)}{\partial z^{2} \partial t^{2}}+\rho A \frac{\partial^{2} v_{1}(z, t)}{\partial t^{2}}=0, \\
0<z<\gamma_{1} L, \\
\mathrm{EI} \frac{\partial^{4} v_{2}(z, t)}{\partial z^{4}}-\mu^{2} \rho A \frac{\partial^{4} v_{2}(z, t)}{\partial z^{2} \partial t^{2}}+(\rho A+\bar{m}) \frac{\partial^{2} v_{2}(z, t)}{\partial t^{2}}=0, \\
\gamma_{1} L<z<\gamma_{2} L, \\
\mathrm{EI} \frac{\partial^{4} v_{3}(z, t)}{\partial z^{4}}-\mu^{2} \rho A \frac{\partial^{4} v_{3}(z, t)}{\partial z^{2} \partial t^{2}}+\rho A \frac{\partial^{2} v_{3}(z, t)}{\partial t^{2}}=0, \\
\gamma_{2} L<z<L,
\end{gathered}
$$

whereas the boundary conditions can be deduced from (A.7),

$$
\begin{aligned}
v_{1}(0, t) & =0, \\
\frac{\partial v_{1}(0, t)}{\partial z} & =0,
\end{aligned}
$$

for $z=0$ and for $z=L$,

$$
\begin{gathered}
\operatorname{EI} \frac{\partial^{3} v_{3}(L, t)}{\partial z^{3}}-\mu^{2} \rho A \frac{\partial^{3} v_{3}(L, t)}{\partial t^{2} \partial z}=0, \\
-\mathrm{EI} \frac{\partial^{2} v_{3}(L, t)}{\partial z^{2}}+\mu^{2} \rho A \frac{\partial^{2} v_{3}(L, t)}{\partial t^{2}}=0 .
\end{gathered}
$$

The boundary conditions for $z=\gamma_{1} L$ are

$$
\begin{gathered}
v_{1}\left(\gamma_{1} L, t\right)=v_{2}\left(\gamma_{1} L, t\right), \\
\frac{\partial v_{1}\left(\gamma_{1} L, t\right)}{\partial z}=\frac{\partial v_{2}\left(\gamma_{1} L, t\right)}{\partial z}, \\
\mu^{2} \rho A \frac{\partial^{3} v_{1}\left(\gamma_{1} L, t\right)}{\partial t^{2} \partial z}-\mathrm{EI} \frac{\partial^{3} v_{1}\left(\gamma_{1} L, t\right)}{\partial z^{3}}-\mu^{2} \rho A \frac{\partial^{3} v_{2}\left(\gamma_{1} L, t\right)}{\partial t^{2} \partial z} \\
+\mathrm{EI} \frac{\partial^{3} v_{2}\left(\gamma_{1} L, t\right)}{\partial z^{3}}=0, \\
\mu^{2} \rho A \frac{\partial^{2} v_{1}\left(\gamma_{1} L, t\right)}{\partial t^{2}}-\mathrm{EI} \frac{\partial^{2} v_{1}\left(\gamma_{1} L, t\right)}{\partial z^{2}}-\mu^{2} \rho A \frac{\partial^{2} v_{2}\left(\gamma_{1} L, t\right)}{\partial t^{2}} \\
+\mathrm{EI} \frac{\partial^{2} v_{2}\left(\gamma_{1} L, t\right)}{\partial z^{2}}=0
\end{gathered}
$$


and those for $z=\gamma_{2} L$ are

$$
\begin{gathered}
v_{2}\left(\gamma_{2} L, t\right)=v_{3}\left(\gamma_{2} L, t\right), \\
\frac{\partial v_{2}\left(\gamma_{2} L, t\right)}{\partial z}=\frac{\partial v_{3}\left(\gamma_{2} L, t\right)}{\partial z}, \\
\mu^{2} \rho A \frac{\partial^{3} v_{2}\left(\gamma_{2} L, t\right)}{\partial t^{2} \partial z}-\mathrm{EI} \frac{\partial^{3} v_{2}\left(\gamma_{2} L, t\right)}{\partial z^{3}}-\mu^{2} \rho A \frac{\partial^{3} v_{3}\left(\gamma_{2} L, t\right)}{\partial t^{2} \partial z} \\
+\mathrm{EI} \frac{\partial^{3} v_{3}\left(\gamma_{2} L, t\right)}{\partial z^{3}}=0, \\
\mu^{2} \rho A \frac{\partial^{2} v_{2}\left(\gamma_{2} L, t\right)}{\partial t^{2}}-\mathrm{EI} \frac{\partial^{2} v_{2}\left(\gamma_{2} L, t\right)}{\partial z^{2}}-\mu^{2} \rho A \frac{\partial^{2} v_{3}\left(\gamma_{2} L, t\right)}{\partial t^{2}} \\
+\mathrm{EI} \frac{\partial^{2} v_{3}\left(\gamma_{2} L, t\right)}{\partial z^{2}}=0 .
\end{gathered}
$$

\section{Conflict of Interests}

The authors declare that there is no conflict of interests regarding the publication of this paper.

\section{References}

[1] S. Iijima, "Helical microtubules of graphitic carbon," Nature, vol. 354, no. 6348, pp. 56-58, 1991.

[2] J. W. Yan, K. M. Liew, and L. H. He, "Free vibration analysis of single-walled carbon nanotubes using a higher-order gradient theory," Journal of Sound and Vibration, vol. 332, no. 15, pp. 3740-3755, 2013.

[3] A. C. Eringen, "On differential equations of nonlocal elasticity and solutions of screw dislocation and surface waves," Journal of Applied Physics, vol. 54, no. 9, pp. 4703-4710, 1983.

[4] A. C. Eringen, Nonlocal Continuum Field Theories, Springer, New York, NY, USA, 2002.

[5] I. Mehdipour, A. Barari, and G. Domairry, "Application of a cantilevered SWCNT with mass at the tip as a nanomechanical sensor," Computational Materials Science, vol. 50, no. 6, pp. 1830-1833, 2011.

[6] I. Mehdipour and A. Barari, "Why the center-point of bridbed carbon nanotube length is the most mass sensitive location for mass attachment?" Sensors and Actuators A, vol. 126, pp. 117-121, 2011.

[7] D. H. Wu, W. T. Chien, C. S. Chen, and H. H. Chen, "Resonant frequency analysis of fixed-free single-walled carbon nanotubebased mass sensor," Sensors and Actuators A: Physical, vol. 126, no. 1, pp. 117-121, 2006.

[8] R. Chowdhury, S. Adhikari, and J. Mitchell, "Vibrating carbon nanotube based bio-sensors," Physica E: Low-Dimensional Systems and Nanostructures, vol. 42, no. 2, pp. 104-109, 2009.

[9] T. L. Horngn, "Analytical solution of vibration analysis on fixedfree single-walled carbon nanotubed-based mass sensor," Journal of Surface Engineered Materials and Advanced Thecnology, vol. 2, pp. 47-52, 2012.

[10] H.-L. Lee, J.-C. Hsu, and W.-J. Chang, "Frequency shift of carbon-nanotube-based mass sensor using nonlocal elasticity theory," Nanoscale Research Letters, vol. 5, no. 11, pp. 1774-1778, 2010.
[11] M. A. De Rosa and M. Lippiello, "Free vibration analysis of SWCNT using CDM in the presence of nonlocal effect," International Journal of Engineering and Innovative Technology, vol. 4, no. 4, pp. 92-102, 2014.

[12] M. A. De Rosa, C. Franciosi, M. Lippiello, and M. T. Piovan, "Nonlocal frequency analysis of nanosensors with attached distributed biomolecules with different boundary conditions," in Proceedings of the 21st Congress on Numerical Methods and Their Applications, Patagonia, Argentina, September 2014.

[13] L. Meirovitch, Fundamentals of Vibrations, McGraw-Hill, 2001.

[14] M. A. De Rosa and M. Lippiello, "Hamilton principle for SWCNT and a modified approach for nonlocal frequency analysis of nanoscale biosensor," International Journal of Recent Scientific Research, vol. 6, no. 1, 2015.

[15] S. Adhikari and R. Chowdhury, "The calibration of carbon nanotube based bionanosensors," Journal of Applied Physics, vol. 107, no. 12, Article ID 124322, 2010.

[16] J. N. Reddy and S. D. Pang, "Nonlocal continuum theories of beams for the analysis of carbon nanotubes," Journal of Applied Physics, vol. 103, no. 2, Article ID 023511, 2008.

[17] T. Murmu and S. Adhikari, "Nonlocal frequency analysis of nanoscale biosensors," Sensors and Actuators A: Physical, vol. 173, no. 1, pp. 41-48, 2012. 

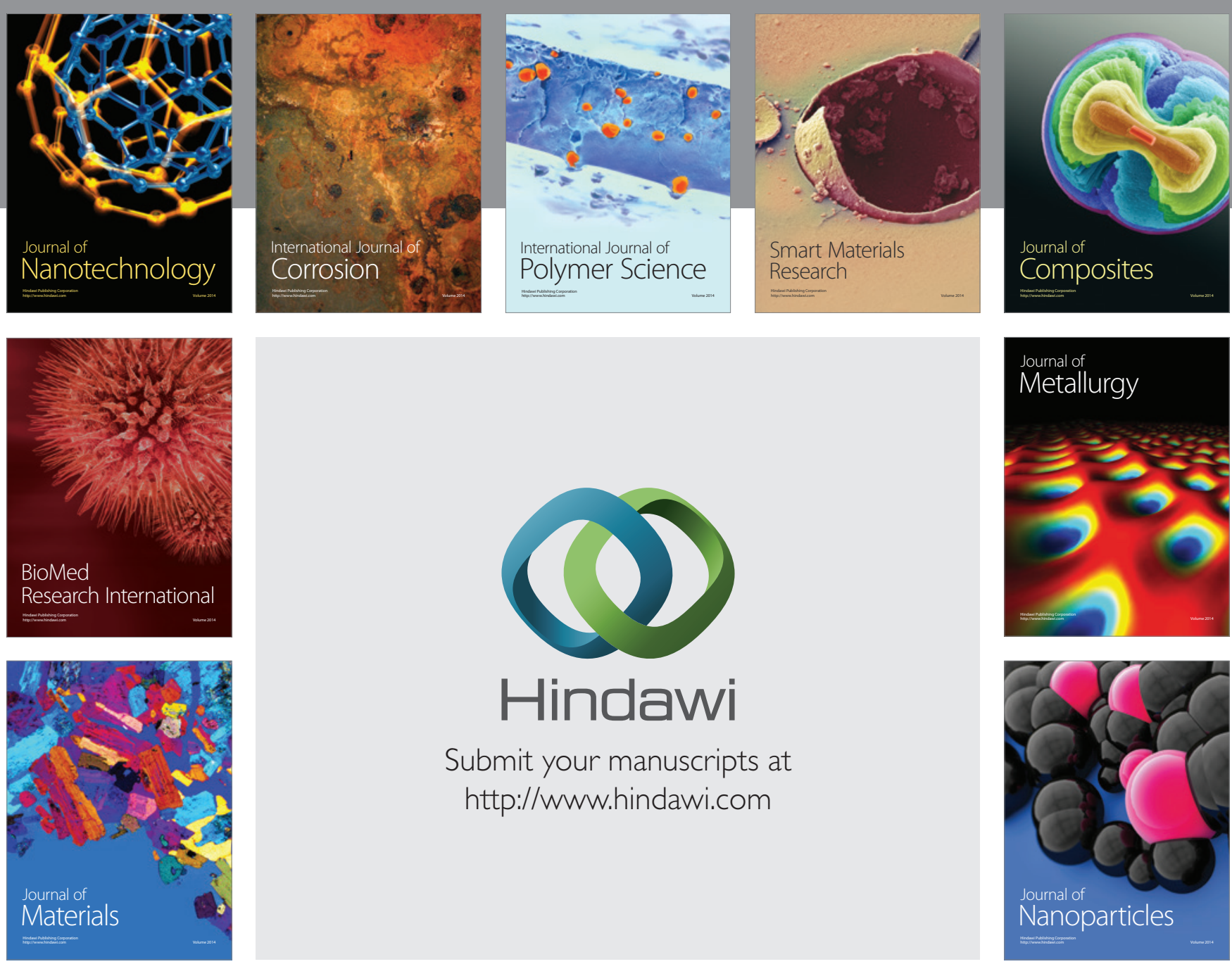

Submit your manuscripts at http://www.hindawi.com
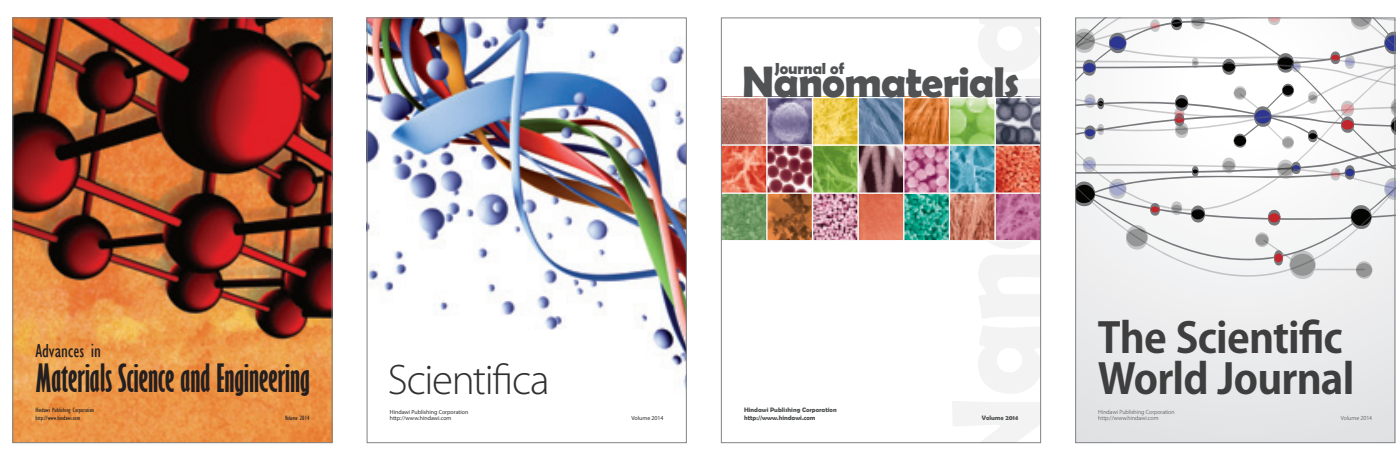

\section{The Scientific World Journal}
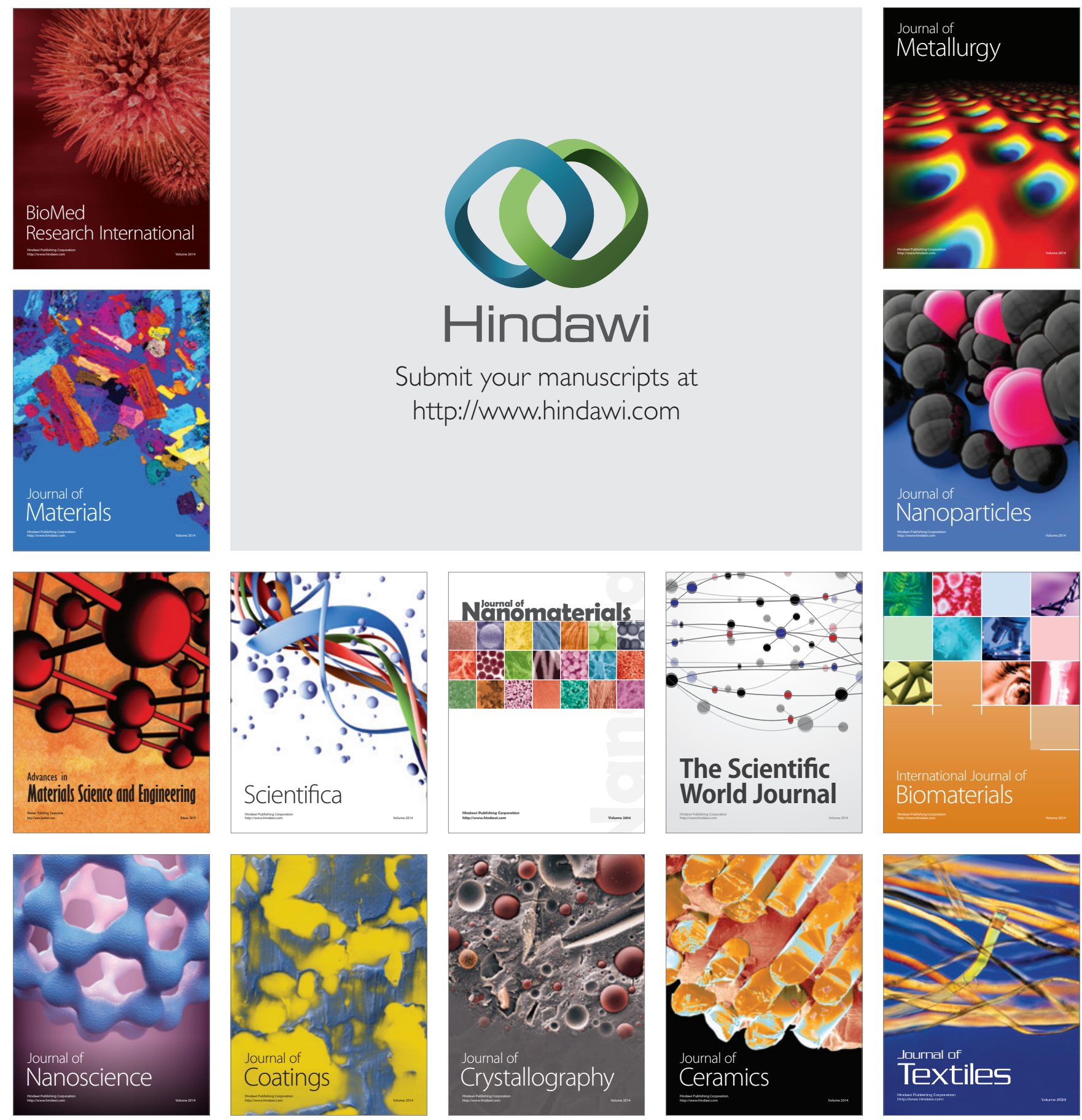